\title{
Research on Robustness Tracking of Maneuvering Target for Bionic Robot
}

\author{
Wang Peng $^{1 *}$, Wu Jian ${ }^{1}$, Zhang Yuan ${ }^{1}$, Li Jixiang ${ }^{2}$ and Zhang Peng ${ }^{2}$ \\ ${ }^{1}$ School of Mechanical and Power Engineering, \\ Harbin University of Science and Technology, Harbin, China \\ ${ }^{2}$ College of Automation, Harbin University of Science and Technology, \\ Harbin, China \\ wangpcw@163.com
}

\begin{abstract}
In order to improve vision tracking quality of the bionic robot, the new automatic tracking algorithm system is proposed in this paper. Base on design of FPGA image acquisition system, the scene noise is removed by adaptive wiener filtering. Aiming at the problem of ROI region extraction in the scene, the seed pixel is selected with background subtraction, orderly, the neighborhood point is judged, the label of the primary selection seed is calibrated. The scene image segmentation algorithm is proposed based on snake model. The matching process is to find the maximum optimization process of the similar function, and the gradient drop method is adopted in mean shift algorithm. Extended kalman filtering is used to realize the robustness state estimation and prediction of the target tracking system. The results given by tracing experiment indicate that the proposed detailed algorithm is effective for partial loss of maneuvering target.
\end{abstract}

Keywords: maneuvering target; tracking algorithm; mean shift; extended kalman filtering

\section{Introduction}

With the tasks growth of explore outer space, build space station, emergency rescue and disaster relief, military reconnaissance under dangerous conditions, anti-terrorist and explosive-handling [1-3], people are not suitable to undertake these activities. We need a large number of corresponding bionic robots which can replace the human [4-6]. Since the new century, the vision target tracking technology has been more applied in the robot. Therefore, the development of the bionic robot with vision function has gradually become the research focus of scholars both at home and abroad.

Moving target detecting and tracking are detecting, extracting and identifying the moving target of video images [7-9], and then obtaining the motion parameters for further analysis and understand the moving target behavior to complete target tracking or further task [10]. The automatic tracking system of maneuvering target is the core of the microprocessor vision system, which fused image processing, pattern recognition, artificial intelligence and automatic control of different fields of advanced interdisciplinary research. It is one of the key technologies to realize intelligent robots and intelligent weapons, which has broad application prospect in the military, transportation, biology, medicine [11-13]. The difficulty of maneuvering target detection and tracking is largely due to the target motion complexity in actual environment and the peculiarity of video data. The whole image size, the speed, the moving trajectory and the stability of background are all to bring difficulty to moving target tracking. ${ }^{1}$

* Corresponding author 
Mean shift is a nonparametric algorithm based on kernel function estimation. It does not need to estimate the probability density of the whole interval, and there is no need to know the distribution of the sample interval. The advantages of mean shift algorithm is low computational complexity, and the fast calculation speed, strong anti-interference ability. In the field of computer vision, it shows good robustness and real-time to solve the target tracking. It finds the target location with the iterations by density gradient ascent. The local maxima of the similarity function is got [14-16]. The maximum point of the image area corresponding is the track target in the current frame position. The application scope of the mean shift algorithm is widely used at home and abroad, a large number of applications is the improved algorithm for literature. The literature [17] put forward the color histogram adaptive update method of hypothesis testing theory based on kalman filtering to solve the model updating problem of mean shift algorithm in the tracking process. But it does not take into account deletion problem of the track target.

In order to improve the vision tracking quality of the bionic robot, and then to improve the practicability completely and expand the application fields, in this paper, we study the bionic robot vision tracking method systematically including wiener filtering, snake contour model, mean shift iteration algorithm, extended kalman filter. So, it can achieve noise elimination, object contour extraction, the robustness of target tracking. The goal is to solve the technical problems that the large instability and the weak real-time produced by bionic robot vision tracking. It ensure that the robot can monitor changes in the work environment.

\section{FPGA Image Acquisition System}

For the tracking system of robot, the main consideration is the problem of the system operation speed and power consumption. The biggest advantage and characteristic of Nios II is the modular hardware structure, and has good ability to modify and extend. This system uses Quartus II as the hardware development platform, using FPGA chip as the controller, the image sensor, memory, video converter, TFT display interface as FPGA peripherals. It is shown in Figure 1.The programming and control of the FPGA and peripherals is with SOPC technology, and eventually realize the image real time collection.

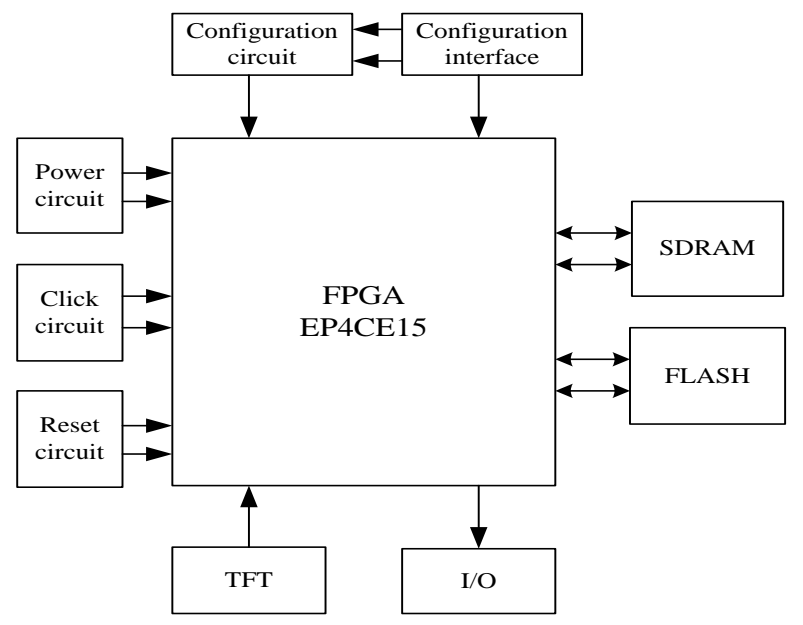

Figure 1. Image Acquisition System Framework

\section{Wiener Filtering Algorithm}

In the obtained images, the target tracking and the background have different statistical characteristics, namely the different mean and variances. Therefore, in 
order to retain the edge information of key target as far as possible, usually, the adaptive local filtering strategy is applied. According to the noise characteristics analyzing of tracking scene, we adopt the adaptive wiener filtering to eliminate noise in this paper. Wiener filter takes image signal and noise as random signal, and the optimality criteria filter will be designed based on the statistical random signal.

Let the signal to be treated compose with useful signal $f(t)$ and additive noise $n(t)$. The transfer function of wiener filter is $h(t)$, its output is $y(t)$. The ideal condition is $y(t)=f(t)$, the transfer function of degradation model is $H_{R}(x, y)$. The degradation model of images can be written as:

$$
g(x, y)=h(x, y) \times f(x, y)+n(x, y)
$$

In order to meet the real-time tracking characteristic of moving robot when the wiener filtering estimation is used to recover images, the restoring matrix $\mathrm{W}$ based on the parameter estimation is used to simplified algorithm implementation in this paper. The image to be recovered is shown as formula (2):

$$
\hat{f}(x, y)=W g(x, y)
$$

With the orthogonal projection theorem, the obtained $\mathrm{W}$ can be shown as:

$W=C_{f g}\left(C_{g g}\right)^{-1}$

Where $C_{g g}$ is the nonsingular covariance matrix, $C_{f g}$ is cross covariance matrix.

Bring them into formula (3), we can get formula (4) and formula (5).

$$
\begin{aligned}
& C_{g g}=H_{R} C_{f} H_{R}^{\prime}+C_{n} \\
& C_{f g}=C_{f} H_{R}^{\prime}
\end{aligned}
$$

Where $C_{f}$ is transition matrix, $C_{n}$ is noise matrix.

The restoring matrix $\mathrm{W}$ can be shown as:

$$
W=C_{f} H_{R}^{\prime}\left(H_{R} C_{f} H_{R}^{\prime}+C_{n}\right)^{-1}
$$

According to the auxiliary theorem of the matrix inversion, we can get formula (7).

$$
W=\left(H_{R}^{\prime} H_{R}+C_{n}\right) H_{R}^{\prime}
$$

Where $C_{n}$ is the sum of all the noise effect in the visual inspection system. Thus, we get the recovery matrix $\mathrm{W}$, and the recovered image sequence is obtained by wiener parameter estimation method.

Wiener filter takes the minimum mean square deviation as optimal criterion to design filter. Even if the target is growing bigger, we also can get satisfactory image detail feature with this algorithm.

\section{Region Growing Algorithm}

The basic thought of region growing is gathering the pixels with similar property to form region. The realizing process is that, taking a seed pixel as starting point for each division region, and then merging the same or similar pixels (judged by the certain growing or similar standard determined in advance) around the field of seed pixel into the seed pixel region. These new pixels are taken as new seed pixels to repeat the process above until all the pixels satisfied the condition have been merged. At that time, one region is grown. After all of the regions are formed, the area of all the connected regions is calculated to test the image, and the region with 
the largest area is taken as the target region. When the region growing method is used in the actual, there are three questions needed to be solved.

Step 1: Determine a group of seed pixels which can represent the needed region correctly.

Step 2: Determine the standard of merging the adjacent pixel during the growing process.

Step 3: Make the condition which can stop the growing process.

Select ROI of seed point.

Step 1: Select the seed pixel with background subtraction. The seed pixel can always be selected with the characteristic of specific problem. In this paper, two arrays are estimated base on the background subtraction. Arrayl is used to save the coordinate value of different pixels of current frame and background, while, Array2 is used to save the lightness value of different pixels. They are sequential storages, the coordinate and the lightness values are corresponding to the same bright point peak.

Step 2: The judging of the neighborhood point. For the label calibrating of the primary selection seed, in this paper, we need to judge whether the four adjacent pixels b, c. d, e are bright points. The processing image size is $640 \times 480$, namely the horizontal line includes 640 pixels. The coordinate values of all the bright points in the Array 1 are counted with the sum of the interval pixels at the image starting point. For example, the 15 th point of the 6 th line whose coordinate value is $640 \times(6-1)+15$. Let the line be $i$, the row be $j$, the coordinate value be $\mathrm{p}$, and the transition is shown as formula (8):

$$
P=640 \times(i-1)+j
$$

According to the coordinate value calibrating, aiming at any point a in the Array1, we can determine whether the four adjacent pixels b, c. d, e are bright points by searching for the bright point whose difference of coordinate value is 639 or 1 .

Step 3: Calibrate the label of the primary selection seed. According to the requirement, adding two labels to each pixel in Array1: one label judges whether the grown label (Array3) is, the other judges whether the marginal label (Array4) is. The two labels are 0 under the initial condition. The growing label is saved in Array3, and the marginal label is saved in Array4. The calibrating of the growing label and the marginal label can be determined by whether the four adjacent pixels b, c. d, e are bright points. If there are two or three bright points, at the point a, the growing label and the marginal label are not 0 , while, If there are four bright points, the growing label is not 0 and the marginal label is 0 . The other conditions, the growing label and the marginal label are 0 .

Step 4: Reduce the range of seed with growing label and the maximal saliency. The point, whose growing label is 0, is eliminated by Array3, refreshing Array1, Array 2 and Array 4 orderly to reduce the range of seed. The eliminated point is noisy point. It has strong filtering action, and the refreshing bright points represent the needed area of seed pixel correctly. The seed point should be with high attention, and it should locate in the object.

\section{Snake Model}

Snake model is a continuous closed curve with minimization energy, and it is inlayed with $\mathrm{n}$ control points. Where $v_{i}$ is the control point after discretization, and $\mathrm{s}$ is arc length. Kass and others constructed proper deformation energy to define the target outline, and then the effect of the variable forces would be shown with proper energy function, when the value reaches the minimum, the active outline approaches the real one. The parameterized representation of the Snake model is shown as $v(s)=(x(s), y(s)), s \in[0,1]$ , which is normalized curve length. The total energy function of the active outline is shown as: 


$$
E_{\text {snake }}=\int_{0}^{1} E(v(s)) d s=\int_{0}^{1}\left[E_{\text {in }}(v(s))+E_{\text {image }}(v(s))+E_{\text {out }}(v(s))\right] d s
$$

The internal energy $E_{i n}$ keeps the snake curve local smoothing, which makes the curve converge to the interest characteristics without enwinding. $v_{s}(s)$ and $v_{s s}(s)$ represents the first derivative and the second derivative respectively. The module value of $v_{s}(s)$ shows the slope of the curve, which controls the continuity. While the module value of $v_{s s}(s)$ shows the curvature, which controls the degree of curve. The energy initiative minimize of $E_{\text {in }}$ makes the curve approach a straight line for the open curve outline.

When the initial outline is got, we need to make the Snake evolution. That means the spline curve moves towards the energy decreasing direction under the action of the internal and the external energy, when it reaches the target edge, $E_{\text {Snake }}$ gets the minimum. In this paper, we analyze the optimization strategy of the energy function minimize algorithm. On the whole, the Greedy algorithm has better effect. We find that the global minimum energy of the dynamic outline can not be gained, and the reason is the strategy of judging the iteration termination can not change the blind search state. In this paper, the greedy strategy combined with genetic algorithm is designed to realize the energy function minimization. The total energy function of Greedy algorithm can be written as:

$$
E=\int\left(a(s) E_{\text {cont }}+\beta(s) E_{\text {curv }}+\gamma(s) E_{\text {image }}\right) d s
$$

To retain the corner during the smooth shrinking process, after the each iteration in the Greedy algorithm, we calculate the curvature of the new position for each control point, if its value satisfies the conditions below:

(1)Local curvature value is the maximum;

(2)The curvature is larger than some closed value;

(3)It is the edge point. $\beta_{i}$ of the control point is zero in the next iteration.

This method feeds back the top-level handling to the energy minimum process, and it can control the local quality during the curve shrinking process better. When the Greedy algorithm stops the iterative, in this paper, we optimize the Greedy evolution result based on the genetic algorithm to gain the final segmentation result with the best track convergence.

\section{The Mean Shift Iteration Algorithm}

The Bhattacharyya coefficient is usually used as the similarity function. The matching process is to find the maximum optimization process of the similar function, and the gradient drop method is adopted in mean shift algorithm. The Taylor expansion is written as formula (11) at $p(x)$ to the similarity function. $\mathrm{x}$ is the center coordinates of the candidate region.

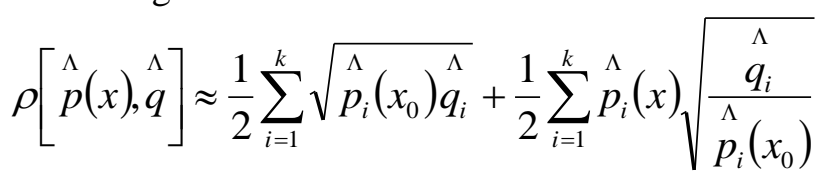

In order to make the $p(x)$ to the maximum, gradient direction for $x_{0}$ is got by the derivative method in formula (12). $\mathrm{L}$ is the bandwidth of kernel function, $\mathrm{G}$ is kernel function, $\mathrm{C}$ is the normalized constant. 


$$
\begin{aligned}
& \nabla \rho[\rho(x), q]=\frac{c_{m} \sum_{i=1}^{n} \omega_{i}\left(x_{0}-x_{i}\right) G\left(\left\|\frac{x_{0}-x_{i}}{l}\right\|^{2}\right)}{l^{2}} \\
& =\frac{c_{m}}{l^{2}} \sum_{i=1}^{n} G\left[\left\|\frac{x_{0}-x_{i}}{l}\right\|\right]^{2}\left[\frac{\sum_{i=1}^{n} \omega_{i} x_{i} G\left[\left\|\frac{x_{0}-x_{i}}{l}\right\|^{2}\right]}{\sum_{i=1}^{n} \omega_{i} G\left[\left\|\frac{x_{0}-x_{i}}{l}\right\|^{2}\right]}-x_{0}\right]
\end{aligned}
$$

In the mean shift iteration time, center of the target area will be shifted from $x_{0}$ to a new location $x_{l}$.

$$
x_{i}=\frac{\sum_{i=1}^{n_{k}} x_{i} \omega_{i} g\left[\left\|\frac{x_{0}-x_{i}}{l}\right\|^{2}\right]}{\sum_{i=1}^{n_{k}} \omega_{i} g\left[\left\|\frac{x_{0}-x_{i}}{l}\right\|^{2}\right]}
$$

Get a new position $x_{i}$, if the distance is $\left\|x_{i}-x_{0}\right\|<\varepsilon$ between the new position and the original position, and read the next frame image. Otherwise, continue to the iterative calculation.

\section{Extended Kalman Filter}

Extended kalman filtering is a method to estimate the linear minimum variance of state variable. It can realize the real-time state estimation and prediction of the target tracking system. When the track target is partially missing, the system still can lock. The state equation and measurement equation can be written as:

$$
\begin{gathered}
x_{t}=f\left(x_{t}, t\right)+w_{t} \\
y_{t}=h\left(x_{t}\right)+v_{t}
\end{gathered}
$$

Where, $x_{t}, y_{t}$ are state estimation of target tracking system, $f$ and $h$ are nonlinear function, $t$ is the number of updates, $w_{t}$ is state disturbance, $v_{t}$ is observation disturbance.

Tracking system must be robust transformation, so:

$$
x_{t}^{\prime}=x_{t}^{\prime}+\Delta x_{t}^{\prime}
$$

Where, $x_{t}^{\prime}$ is estimator, $\Delta x_{t}^{\prime}$ is state correction parameters.

The state estimation can be gained with Taylor series expansion for nonlinear system $\Delta x_{t}^{\prime}$, and an approximate solution is got.

$$
\left\{\begin{array}{l}
x_{t}=f(x, t)\left|x_{t}^{\prime}=x_{t}+\frac{\partial f\left(x_{t}^{\prime} t^{\prime}\right)}{\partial x_{t}}\right| x_{t}^{\prime} \Delta x_{t}+w_{t} \\
y_{t}=h(x, t)\left|x_{t}^{\prime}=x_{t}+\frac{\partial h\left(x_{t}^{\prime} t\right)}{\partial x_{t}}\right| x_{t}^{\prime} \Delta x_{t}+v_{t}
\end{array}\right.
$$


The extended Kalman filter equation is used.

$$
\begin{gathered}
x_{t, t-1}=E_{t, t-1} x_{t-1} \\
x_{t}=x_{t, t-1}+K_{t}\left(y_{t}\right)-H_{t} x_{t, t-1} \\
K_{t}=P_{t} H_{t}^{T} R_{t}^{-1} \\
P_{t, t-1}=E_{t, t-1} P_{t-1} E_{t, t-1}^{T}+Q_{t-1} \\
P_{t}=\left(1-K_{t} H_{t}\right) P_{t, t-1}
\end{gathered}
$$

Where, $x_{t, t-1}$ is the state observation matrix, $E_{t, t-1}$ is state transition matrix, $x_{t-1}$ is the state estimation of t-1 moment, $K_{t}$ is the gain matrix, $H_{t}$ is the observation matrix, $P_{t}$ is the covariance matrix, $P_{t, t-1}$ is the covariance estimation of t-1 moment, $R$ and $Q$ are the system state and the measured variance.

\section{Experiment and Analysis}

Figure 2-4 are the tracking results for maneuvering target. Figure 2 is irregular target tracking. There are a finger and fist in the scene. The background is black and white, there is interference in the color. The traditional rectangular mark is changed, the oval mark is used. It is to facilitate the signposted for ROI area. Posture is inclined, and there is partial deletion of tracking object in Figure 3. The oval mark wrap minimum range with contour. There is only fist in Figure 4. In this case, the target tracking can be firmly locked. From them we can see, tracking results show that the proposed algorithm has good universality and generality, not like using the object simple geometric feature method which needs to select different features for different shape by artificially. That means the improved algorithm still can accurately lock the dynamic target under the condition of that the partial occlusion existing and the outer contour feature of target changing.

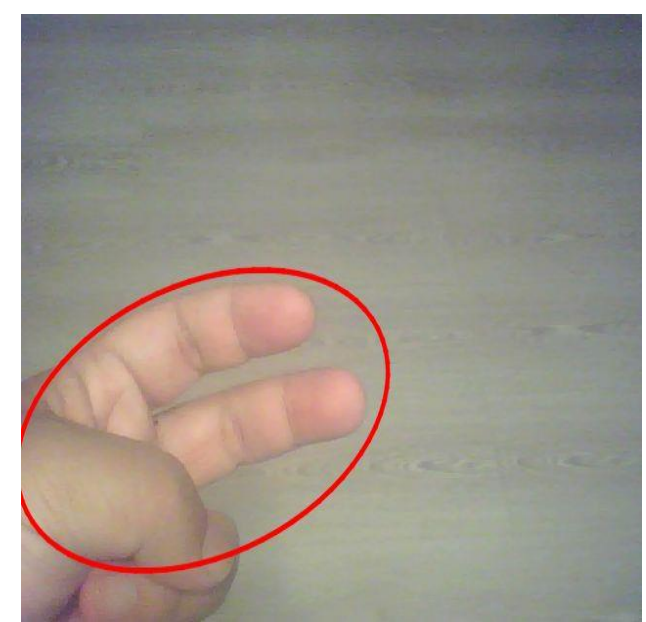

Figure 2. Sequence Experimental Result 1 


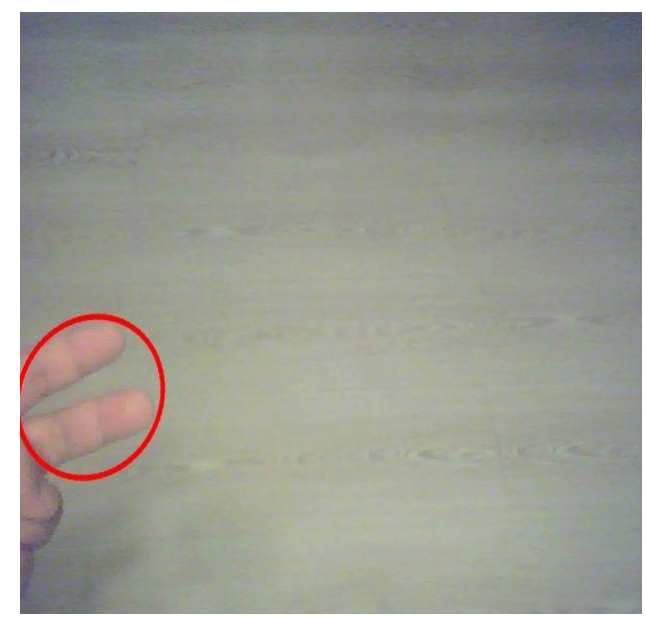

Figure 3. Sequence Experimental Result 2

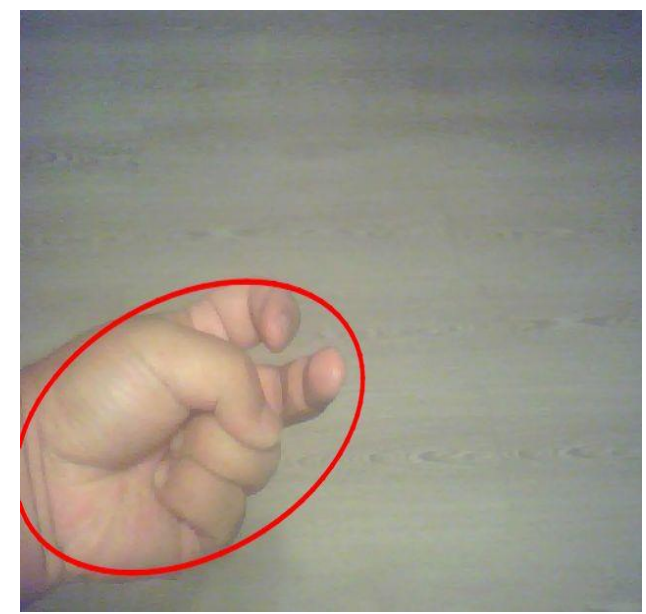

Figure 4. Sequence Experimental Result 3

\section{Conclusions}

Aiming at the problems of stability and real-time existing in the bionic robot visual system, we put forward a new algorithm of maneuvering target tracking system in this paper. According to scene characteristics, the scene noise is removed by adaptive wiener filtering. The unified of the feature region is realized, namely ROI extraction based on the region growing. The scene image segmentation algorithm is proposed based on snake model. The robustness state estimation and prediction is realized for the target tracking system with mean shift algorithm and extended kalman filtering. Tracking experiment results analysis shows that, this algorithm system has strong noise resisting ability, high locking precision and strong adaptability to complete automatic tracking.

\section{Acknowledgments}

This work was supported by National Natural Science Foundation of China (Grant NO. 51105122).

\section{References}

[1] P. Wang, Y. Zhang and J.L. Xin, "Research Vision-Guided Robot for Obstacle Avoidance of Information Fusion in the Unstructured Environment", Sensor Letters, vol. 9, no. 5, (2011), pp. 20212024. 
[2] D. Jia, L.F. Zhang and C. L. H. Wolswijk, "The Improvement of Mean-Shift Algorithm in Target Tracking," International Journal of Security and Its Applications, vol. 9, no. 2, (2015), pp. 21-28.

[3] A. Yilmaz, O. Javed and M. Shah , "Object Tracking: a Survey," ACM Computing Surveys, vol. 38, no. 4, (2006), pp. 1-45.

[4] M. Salzmann, J. Pilet, S. Llic and P. Fua, "Surface Deformation Models for Nonrigid 3D Shape Recovery", IEEE Transactions on Pattern Analysis and Machine Intelligence, vol. 29, no. 8, (2007), pp. 1481-1487.

[5] G.R. J and G.C. M, "Surveillance and Human-computer Interaction Applications of Self-growing Models", Applied Soft Computing, vol. 11, no.7, (2011), pp. 4413- 4431.

[6] S.C. Chan, S. Zhang and J. F. Wu, "On the Hardware/Software Design and Implementation of a High Definition Multiview Video Surveillance System", Journal on Emerging and Selected Topics in Circuits and Systems, vol. 3, no. 2, (2013), pp. 248-260.

[7] M. C. Roh, T. Y. Kim and J. Park, "Accurate Object Contour Tracking Based on Boundary Edge Selection”, Pattern Recognition, vol. 40, no. 3, (2007), pp. 931-943.

[8] B.F. Francisco, O. Alberto and O. Gabriel, "Visual Navigation for Mobile Robots: A Survey", Journal of Intelligent and Robotic Systems, vol. 53, no. 3, (2008), pp. 263- 296.

[9] H. Fujiyoshi and T. Kanade, "VSAM:Video Surveillance and Monitoring Project", Journal of the Institute of Image Infornation and Television Engineers, vol. 57, no. 9, (2003), pp. 1068-1072.

[10] D. Comaniciu , V. Ramesh and P. Meer , "Kernel-based Object Tracking", IEEE Transactions on Pattern Analysis and Machine Intelligence, vol. 25 no. 2, (2003), pp. 564- 577.

[11] M. Robin, "Human-robot Interaction in Rescue Robotics", IEEE Transactions on Systems, Man and Cybernetics Part C: Applications and Reviews, Human-Robot Interactions, vol. 34, no. 2, (2004), pp. 138-153.

[12] R.T. Collins, Y. Liu and M. Leordeanu, “Online Selection of Discriminative Tracking Features,” IEEE Transactions on Pattern Analysis and Machine Intelligence, vol. 27, no. 10, (2005), pp. 1631- 1643.

[13] D. Ross, J. Lim, R. Lin and M. Yang , "Incremental Learning for Robust Visual Tracking", International Journal of Computer Vision, vol. 77, no. 1-3, (2008), pp. 125- 141

[14] D. Ross, D. Tarlow and R. Zemel, "Learning Articulated Structure and Motion", International Journal of Computer Vision, vol. 88, no. 2, (2010), pp. 214- 237.

[15] P. Pan and D. Schonfeld, "Video Tracking Based on Sequential Particle Filtering on Graphs", IEEE Transactions on Image Processing, vol. 20, no. 6, (2011), pp. 1641-1651.

[16] M. F. Talu, I. Turkoglu and M. Cebeci, "A Hybrid Tracking Method for Scaled and Oriented Objects in Crowded Scenes", Expert Systems with Applications, vol. 38, no. 11, (2011), pp. 13682- 13687.

[17] N. S Peng and J. Yang, "Mean Shift Blob Tracking with Kernel Histogram Filtering and Hypothesis Testing", Pattern Recognition Letters, vol. 26, no. 5, (2005), pp. 605-614.

\section{Authors}

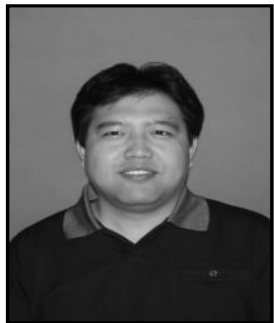

Wang Peng, Male, He was born in Harbin, China, in 1976. He received the M.S. degree, in 2005, and Ph.D. degree, in 2008, from Harbin University of Science and Technology, (China). He was a Postdoctoral at bionic robot lab, College of Automation, Harbin Engineering University (China), in 2009-2011. He is currently a professor at School of Mechanical and Power Engineering, Harbin University of Science and Technology. His research interests include bionic robotics, medical robotics, robot vision, the control of robotic mechanism, and mobile robot.

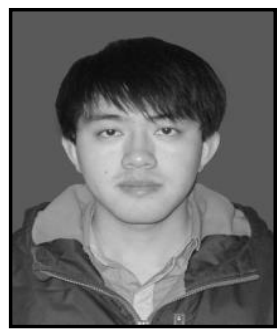

Wu Jian, Male, He was born in Harbin, China, in 1990. He received the B.S. degree, in $2013 \mathrm{He}$ is currently a master degree candidate at School of Mechanical and Power Engineering, Harbin University of Science and Technology. His research interests include bionic robotics, the control of robotic mechanism, and mobile robot. 
International Journal of Security and Its Applications

Vol.9, No.12 (2015) 